\title{
4-2014
}

\section{Magnet Traveling through a Conducting Pipe: A Variation on the Analytical Approach}

\author{
Benjamin Irvine \\ birvine@luc.edu \\ Matthew Kemnetz \\ mkemnetz@luc.edu \\ Asim Gangopadhyaya \\ Loyola University Chicago, agangop@luc.edu \\ Thomas Ruubel \\ Loyola University Chicago, truubel@luc.edu
}

Follow this and additional works at: https://ecommons.luc.edu/physics_facpubs

Part of the Physics Commons

\section{Recommended Citation \\ Irvine, Benjamin, Matthew Kemnetz, Asim Gangopadhyaya, and Thomas Ruubel. "Magnet Traveling through a Conducting Pipe: A Variation on the Analytical Approach." American Journal of Physics 82, no. 4 (April 2014): 273-79. doi:10.1119/1.4864278.}

This Article is brought to you for free and open access by the Faculty Publications and Other Works by Department at Loyola eCommons. It has been accepted for inclusion in Physics: Faculty Publications and Other Works by an authorized administrator of Loyola eCommons. For more information, please contact ecommons@luc.edu. Copyright (c) 2014 American Institute of Physics. This article may be downloaded for personal use only. Any other use requires prior permission of the author and the American Institute of Physics. The following article appeared in Irvine, Benjamin, Matthew Kemnetz, Asim Gangopadhyaya, and Thomas Ruubel. "Magnet Traveling through a Conducting Pipe: A Variation on the Analytical Approach." American Journal of Physics 82, no. 4 (April 2014): 273-79. doi:10.1119/1.4864278 and may be found at http://dx.doi.org/10.1119/1.4864278. 


\section{AAPT AMERICAN \\ of PHYSICS}

\section{Magnet traveling through a conducting pipe: A variation on the analytical approach}

Benjamin Irvine, Matthew Kemnetz, Asim Gangopadhyaya, and Thomas Ruubel

Citation: American Journal of Physics 82, 273 (2014); doi: 10.1119/1.4864278

View online: http://dx.doi.org/10.1119/1.4864278

View Table of Contents: http://scitation.aip.org/content/aapt/journal/ajp/82/4?ver=pdfcov

Published by the American Association of Physics Teachers

\section{Articles you may be interested in}

Electromagnetic Induction with Neodymium Magnets

Phys. Teach. 51, 344 (2013); 10.1119/1.4818369

Magnetic damping: Integrating experimental and theoretical analysis

Am. J. Phys. 80, 27 (2012); 10.1119/1.3647997

Damped fall of magnets inside a conducting pipe

Am. J. Phys. 79, 193 (2011); 10.1119/1.3531964

Laboratory exercises on oscillation modes of pipes

Am. J. Phys. 77, 204 (2009); 10.1119/1.3050179

Investigation of magnetic damping on an air track

Am. J. Phys. 74, 974 (2006); 10.1119/1.2232645

\section{WebAssign.}

Free Physics Videos

Add these videos and many more

resources - free with WebAssign.

bit.do/PhysicsResources

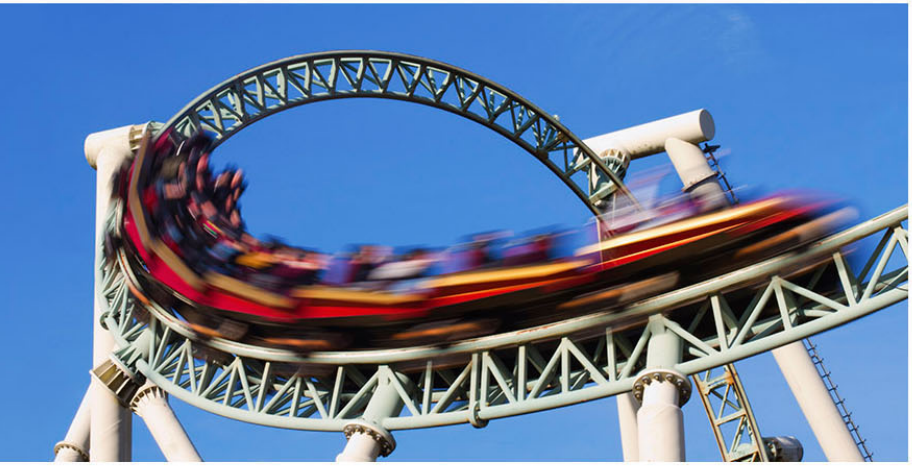




\title{
Magnet traveling through a conducting pipe: A variation on the analytical approach
}

\author{
Benjamin Irvine, ${ }^{\text {a) }}$ Matthew Kemnetz, ${ }^{\text {b) }}$ Asim Gangopadhyaya, ${ }^{c)}$ and Thomas Ruubel ${ }^{\text {d) }}$ \\ Department of Physics, Loyola University Chicago, Chicago, Illinois 60626
}

\begin{abstract}
(Received 15 October 2012; accepted 24 January 2014)
\end{abstract}

\begin{abstract}
We present an analytical study of magnetic damping. In particular, we investigate the dynamics of a cylindrical neodymium magnet as it moves through a conducting tube. Owing to the very high degree of uniformity of the magnetization for neodymium magnets, we are able to provide completely analytical results for the electromotive force generated in the pipe and the consequent retarding force. Our analytical expressions are shown to have excellent agreement with experimental observations. (C) 2014 American Association of Physics Teachers.
\end{abstract}

[http://dx.doi.org/10.1119/1.4864278]

\section{INTRODUCTION}

Magnetic braking plays an increasingly significant role in industry. At present, it is commonly used in applications such as electric vehicles, rowing machines, roller coasters and freefall tower rides in amusement parks, Maglev trains, etc. Other emerging applications include high-speed trains and military vehicles and systems. Magnetic braking has not yet achieved its full potential use in industrial and transportation applications, even though the first patent for a magnetic brake was issued in 1892. There are a number of promising applications in the mining, railroad, and elevator industries, where currently used mechanical brake systems are prone to overheating, mechanical degradation, significant maintenance costs, and periodic failures. Besides being wear free, magnetic brakes are also quiet and odorless. Because they are not dependent on the coefficient of friction, relatively large braking forces can be achieved independent of weather conditions. Furthermore, because their retarding force is proportional to the velocity, they possess an almost ideal mechanism for high-speed safety braking. An improved understanding of magnetic damping is important to the development of future magnetic braking technology. In industry, complex computational models are often used to simulate realistic scenarios of magnetic braking. Here, we have developed a fully theoretical model for a cylindrically symmetric system, which can be used to benchmark these computational models.

We present an analysis of a common demonstration that comprises a cylindrical magnet and a non-ferromagnetic conducting tube in relative motion to each other. ${ }^{1-21}$ Owing to the interaction between the moving magnet and the induced current in the pipe, the magnet falls quite slowly through the tube, and generates a sense of amazement in students and teachers alike. In this paper, we specifically study the motion of a cylindrical neodymium magnet through a copper pipe of circular cross-section. The azimuthal symmetry of the problem keeps the mathematics tractable and allows us to generate an analytical expression for the electromotive force (emf) generated in an arbitrary segment of the tube, as well as the resulting retarding force.

This paper is organized as follows. In Sec. II, we describe the experimental setup used for the demonstration. In Sec. III, we develop a model based on the near-uniformity of magnetization of neodymium magnets and show that the resulting prediction of the magnetic field strength has excellent agreement with the measured values of the field on the axis of the magnet. We also compare the experimental results with the common point-dipole approximation and with a two-monopole approximation. In Sec. IV, we use our model to compute the flux through circular loops of the conducting pipe and generate an expression for the current in a section of pipe of arbitrary length. Then, assuming the length of the tube to be much larger than that of the magnet, in Sec. V we compute the current generated in the pipe. In Sec. VI, we compute the force on the magnet due to the interaction between the magnet and the pipe and compare it with experimental results.

\section{EXPERIMENTAL SETUP}

The experimental setup is shown in Fig. 1; we use two hanging masses ( $m$ and $M$ ) to pull a cylindrical neodymium magnet through a copper pipe with varying terminal velocities. Pulling on the magnet from both sides helps to keep it vertical and prevents it from trying to align with the geomagnetic field. We use "smart pulleys" from PASCO to record the position, velocity, and acceleration of the magnet as it

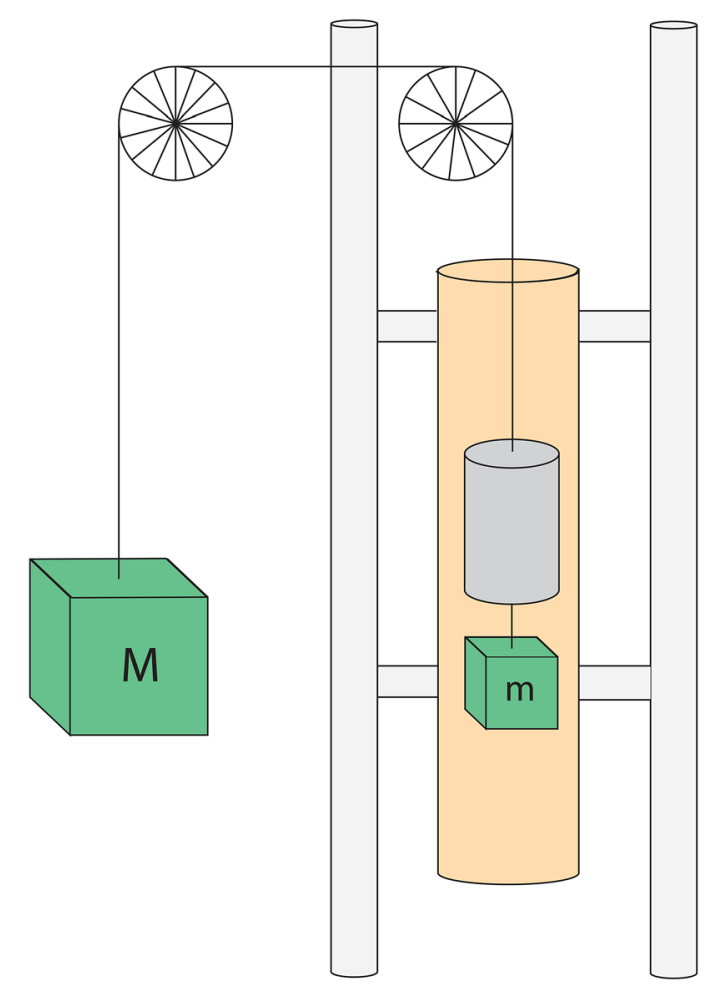

Fig. 1. Schematic of the experimental setup. A two-mass pulley system is used to pull a neodymium magnet through a copper pipe. 


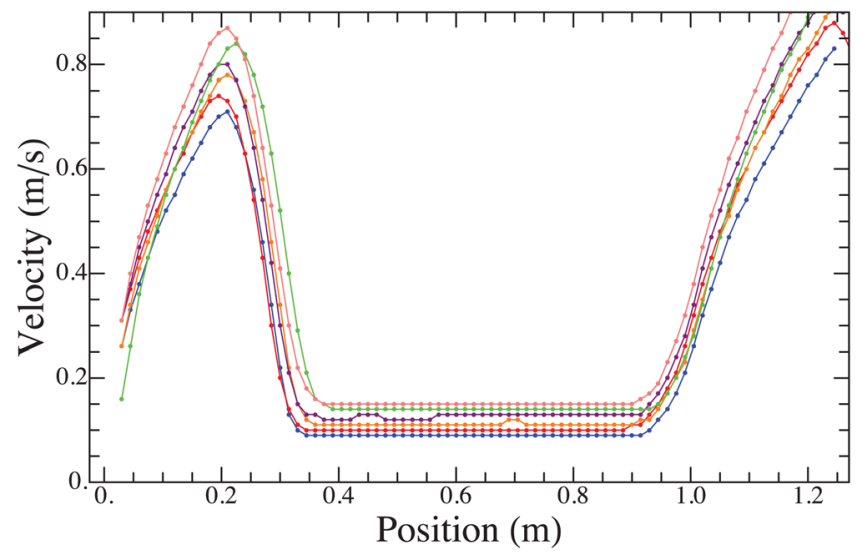

Fig. 2. The velocity of the magnet as it travels into, through, and out of the conducting pipe for different applied forces. The velocity is seen to be constant from around $0.4-0.9 \mathrm{~m}$.

travels into, through, and out of the pipe. Figure 2 shows that the velocity of the magnet remains constant (at the terminal velocity) for a significant segment of the trajectory.

We find that the dependence of the retarding force on the terminal velocity can be accurately modeled by a linear relation. As we will show in Sec. VI, this linear relationship is replicated by our theoretical analysis. Although researchers have studied the damped oscillatory motion of a magnet in a conducting tube, ${ }^{21}$ in this work, we limit ourselves to a study of the retarding force for a magnet moving with different terminal velocities.

\section{MAGNETIC FIELD DUE TO A NEODYMIUM MAGNET}

In order to quantitatively express the magnetic field, we need to develop an appropriate model of the magnet. Several authors have considered the magnet to be a pointdipole, ${ }^{5,15-17,21}$ which works well for small magnets moving through large-diameter pipes (so long as the magnet is not too close to the inner surface of the pipe). Some authors have also considered a physical dipole constructed of two point monopoles separated by an appropriate distance; ${ }^{4}$ this too would be a good approximation when the diameter of the magnet is much smaller than the diameter of the pipe. Our aim here is to keep the analysis as general as possible. In particular, we specifically include the case where the diameters of the magnet and the inner surface are comparable so that a strong braking force is generated. In such a case, the pointdipole model does not accurately fit the experimental data.

Neodymium magnets have a very uniform magnetization. This uniformity allows us to approximate the $\vec{B}$-field of the cylindrical magnet as if it were produced by two circular disks with uniform magnetic surface charge densities $\pm \sigma_{m}$, where $\sigma_{m}$ is equal to the magnetization $M_{0}$ of the magnet. ${ }^{23}$ The method of determining the $\vec{H}$-field is then analogous to the case of finding the electric field of a circular, parallel-plate capacitor with uniform surface charge densities $\pm \sigma_{e}$. In Ref. 4, although the authors recognized the applicability of the two-disk model for this situation, they later chose to approximate it using a two-monopole system.

\section{A. Magnetism in a polarizable medium}

The magnetic field due to a current density $\vec{J}$ must satisfy Ampere's Law

$$
\vec{\nabla} \times \vec{B}=\mu_{0} \vec{J}
$$

where $\vec{J}=\vec{J}_{f}+\vec{J}_{b}$ includes both free and bound current, and the bound current is given by $\vec{J}_{b}=\vec{\nabla} \times \vec{M}$, with $\vec{M}$ the magnetization (magnetic moment per unit volume). Thus, in the presence of magnetization, we have

$$
\vec{\nabla} \times \vec{B}=\mu_{0}\left(\vec{J}_{f}+\vec{J}_{b}\right)=\mu_{0}\left(\vec{J}_{f}+\vec{\nabla} \times \vec{M}\right) .
$$

But for a permanent magnet $\overrightarrow{J_{f}}=0$, so Eq. (2) gives

$$
\vec{\nabla} \times\left(\vec{B}-\mu_{0} \vec{M}\right)=\vec{\nabla} \times \mu_{0} \vec{H}=0,
$$

where the conservative field $\vec{H}$ is defined such that $\vec{B}$ $=\mu_{0}(\vec{M}+\vec{H})$. Further, because $\vec{\nabla} \cdot \vec{B}=0$ we have

$$
\vec{\nabla} \cdot \vec{H}=-\vec{\nabla} \cdot \vec{M}
$$

Comparing this equation with Gauss' law $\vec{\nabla} \cdot \vec{E}=\rho_{e} / \epsilon_{0}$, we see that the $\vec{H}$-field is generated by the "magnetic charge density" $\rho_{m} \equiv-\vec{\nabla} \cdot \vec{M}$ in exactly the same way that the electrostatic field $\vec{E}$ is generated by the electrical charge density $\rho_{e}$. This means we can calculate $\vec{H}$ exactly as we would calculate $\vec{E}$ for an electrostatics problem.

\section{B. Magnetic scalar potential for a uniformly magnetized cylinder}

Because $\vec{H}$ is a conservative field, we can write $\vec{H}=-\vec{\nabla} \Psi_{m}$, where $\Psi_{m}$ is the magnetic scalar potential. ${ }^{23}$ Using Eq. (4), we then see that the magnetic scalar potential obeys Laplace's equation

$$
\vec{\nabla}^{2} \Psi_{m}=-\rho_{m}=\vec{\nabla} \cdot \vec{M}
$$

For a cylindrical magnet of radius $R_{m}$ and length $L$ and having uniform magnetization $M_{0} \hat{e}_{z}$ (along the axis of the cylinder), the divergence is zero everywhere inside (and outside) the magnet and receives non-zero contributions only at the two circular end surfaces. Use of the divergence theorem then shows that the $\vec{H}$-field generated by the cylindrical magnet is the same as that generated by the surface charge density $\hat{n} \cdot \vec{M}$, where $\hat{n}$ is the outward-pointing normal to the cylindrical surface. Physically, this corresponds to two disks of uniform magnetic surface charge densities $\pm \sigma_{m}$ separated by a distance $L$, where $\sigma_{m}=M_{0}$ (see Fig. 3). This expression for the $\vec{H}$-field is valid both inside and outside the magnet. The $\vec{B}$-field is then given by $\mu_{0} \vec{H}$ outside the magnet and $\mu_{0}(\vec{H}+\vec{M})$ inside.

Because of the symmetry of the problem, the scalar potential on the axis of the cylinder is particularly straightforward to calculate. If we begin with a circular disk of radius $R_{m}$ and

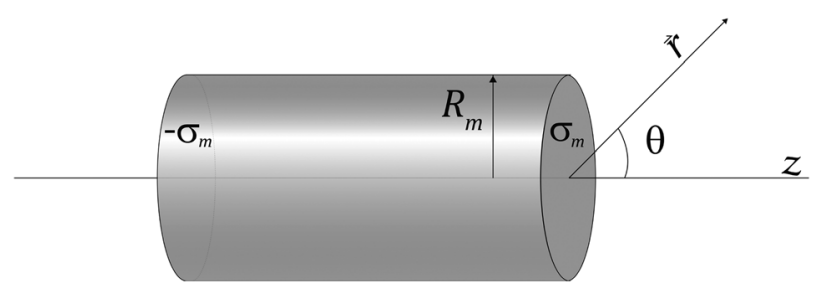

Fig. 3. A cylindrical (neodymium) magnet is modeled as two disks with uniform magnetic charge densities $\pm \sigma_{m}$ located at $z= \pm L / 2$, respectively. 
uniform (magnetic) charge density $M_{0}$ centered at the origin in the $x y$-plane, then the (magnetic) scalar potential on the $z$-axis is given by

$$
\Psi_{m}(z)=\frac{M_{0}}{2}\left(\sqrt{R_{m}^{2}+z^{2}}-z\right) .
$$

To get the scalar potential for the uniformly magnetized cylinder, we use Eq. (6) and the following superposition. If the origin is located at the center of the magnet and the ends of the cylinder are located at $z= \pm L / 2$, as shown in Fig. 3, the resulting scalar potential $\Psi_{m}$ is given by

$$
\begin{aligned}
\Psi_{m}= & \frac{M_{0}}{2}\left\{\left[\sqrt{R_{m}^{2}+\left(z-\frac{L}{2}\right)^{2}}-\left(z-\frac{L}{2}\right)\right]\right. \\
& -\left[\sqrt{\left.\left.R_{m}^{2}+\left(z+\frac{L}{2}\right)^{2}-\left(z+\frac{L}{2}\right)\right]\right\}}\right. \\
= & \frac{M_{0}}{2}\left[\sqrt{R_{m}^{2}+\left(z-\frac{L}{2}\right)^{2}}-\sqrt{R_{m}^{2}+\left(z+\frac{L}{2}\right)^{2}}+L\right] .
\end{aligned}
$$

The magnetic field outside the magnet and along the $z$-axis is then given by

$$
\begin{aligned}
B_{z} & =\mu_{0} H_{z}=-\mu_{0} \frac{\partial \Psi_{m}}{\partial z} \\
& =\frac{\mu_{0} M_{0}}{2} \frac{\partial}{\partial z}\left[\sqrt{R_{m}^{2}+\left(z+\frac{L}{2}\right)^{2}}-\sqrt{R_{m}^{2}+\left(z-\frac{L}{2}\right)^{2}}\right] .
\end{aligned}
$$

To determine $M_{0}$ we substitute the physical dimensions of the magnet $\left(L=5.1 \mathrm{~cm}\right.$ and $\left.R_{m}=1.27 \mathrm{~cm}\right)$ into Eq. (8) and compare it with the experimental data for the axial magnetic field (see Fig. 4). Using the curvefit program in Mathematica we find $M_{0}=1.0335 \times 10^{6} \mathrm{~A} / \mathrm{m}$, which gives a magnetic dipole moment of $\pi R_{m}^{2} L M_{0}=27 \mathrm{~A} \mathrm{~m}^{2}$. Also shown in this

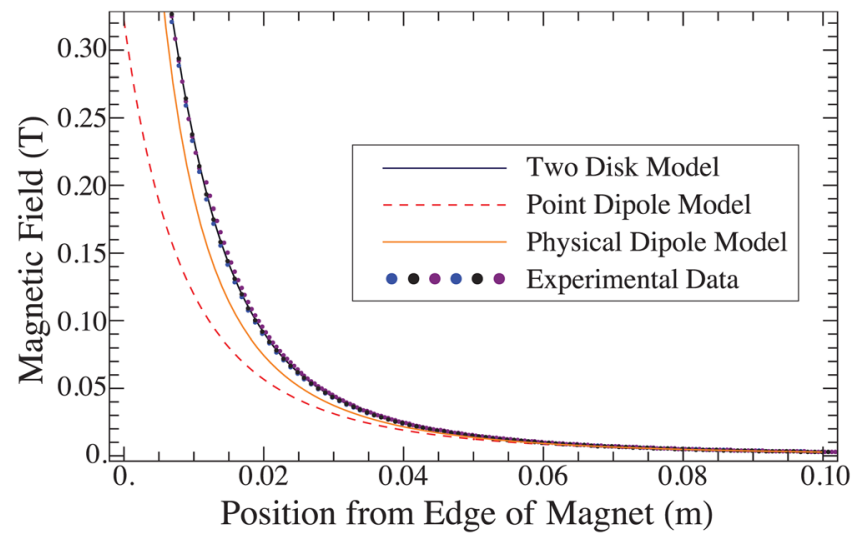

Fig. 4. Axial magnetic field for a point-dipole model (dashed), a physical dipole (gray solid), the Two-Disk model (black solid) and the experimental data (dots); position 0 corresponds to the surface of the magnet. The magnetic field data was collected using a Lakeshore Gaussmeter (model \#460) mounted on a linear translation stage. figure are the predictions of the point-dipole and two-monopole models. ${ }^{22}$ As is evident from Fig. 4, the experimental data are in excellent agreement with the predictions of the two-disk model, which verifies our assumption of uniform magnetization. (It is interesting to note that the two-monopole graph, with magnetic moment $m=27 \mathrm{~A} \mathrm{~m}^{2}$ and length $\ell_{m}=3.245 \mathrm{~cm}$ fits the experimental data surprisingly well.)

\section{Magnetic field of a cylindrical magnet}

To compute the off-axis magnetic field, we begin with the axial potential given in Eq. (6). Except for points on one of the circular end surfaces of the magnet, the magnetic scalar potential $\Psi_{m}$ satisfies $\nabla^{2} \Psi_{m}=0$. Hence, the general solution for $\Psi_{m}$ due to a single disk is given, in spherical coordinates, by

$$
\Psi_{m}(r, \theta)=\sum_{\ell=0}^{\infty}\left(a_{\ell} r^{\ell}+\frac{b_{\ell}}{r^{\ell+1}}\right) \mathcal{P}_{\ell}(\cos \theta),
$$

where $\mathcal{P}_{\ell}(\cos \theta)$ are Legendre Polynomials ${ }^{24}$ in $\cos \theta$.

As we will see, for the calculation of flux we only need to work in the region $r>R_{m}$. Hence, $a_{\ell}=0$ for all $\ell$ and the scalar potential reduces to

$$
\Psi_{m}(r, \theta)=\sum_{\ell=0}^{\infty} \frac{b_{\ell}}{r^{\ell+1}} \mathcal{P}_{\ell}(\cos \theta) .
$$

In order to determine the $b_{\ell}$ 's, we note that the expression for $\Psi_{m}(r, \theta)$ in Eq. (10) must be equal to $\Psi_{m}(z)$ in Eq. (6) when $\theta=0$ (and hence $r=z$ ). Therefore,

$$
\sum_{\ell=0}^{\infty}\left(\frac{b_{\ell}}{r^{\ell+1}}\right)=\frac{M_{0}}{2}\left[\sqrt{R_{m}^{2}+r^{2}}-r\right],
$$

where we have used the fact that $\mathcal{P}_{\ell}(1)=1$ for all $\ell$. By comparing the powers of $r$ on both sides of Eq. (11), we find that the odd coefficients $b_{2 \ell+1}$ are zero, and the even coefficients $b_{2 \ell}$ are given by

$$
b_{2 \ell}=\left[\frac{M_{0} R_{m}^{2 \ell+2}}{2(\ell+1) !}\right] \prod_{k=0}^{\ell}\left(\frac{1}{2}-k\right) .
$$

Thus, the magnetic scalar potential $\Psi_{m}(r, \theta)$, generated by one disk, is given by

$$
\begin{aligned}
\Psi_{m}(r, \theta) & =\sum_{\ell=0}^{\infty}\left[\frac{M_{0} R_{m}^{2 \ell+2}}{2(\ell+1) !}\right] \frac{\prod_{k=0}^{\ell}\left(\frac{1}{2}-k\right)}{r^{2 \ell+1}} \mathcal{P}_{2 \ell}(\cos \theta) \\
& =\frac{M_{0} R_{m}}{2} \sum_{\ell=0}^{\infty} \frac{\prod_{k=0}^{\ell}\left(\frac{1}{2}-k\right)}{(\ell+1) !}\left(\frac{R_{m}}{r}\right)^{2 \ell+1} \mathcal{P}_{2 \ell}(\cos \theta) .
\end{aligned}
$$

Similar to the axial situation [see Eqs. (7) and (8)], to determine the magnetic field of the cylindrical magnet at an arbitrary point $(r, \theta)$, we will again need to add the scalar potentials generated by two such disks with charge densities $\pm M_{0}$ located at positions $z= \pm L / 2$. Hereafter, $\Psi_{m}(r, \theta)$ will 
refer to the scalar potential of the entire magnet. In terms of this re-defined $\Psi_{m}(r, \theta)$, the magnetic field $\vec{B}$ outside the magnet $(|z|>L / 2)$ is given by

$$
\vec{B}=-\mu_{0} \vec{\nabla} \Psi_{m}
$$

while for any point inside the magnet $(|z|<L / 2)$, we have

$$
\vec{B}=-\mu_{0}\left(\vec{\nabla} \Psi_{m}-\vec{M}\right) .
$$

Thus, we have found an exact expression for the magnetic field (outside a distance $R_{m}$ ). The sum can be computed to any desired level of accuracy by including a sufficiently large number of terms. Partovi et al. ${ }^{14}$ have carried out a comprehensive analysis for a uniformly magnetized cylinder falling in a pipe by considering the vector potential due to the moving magnet. Similarly, Derby et al. ${ }^{19}$ computed the magnetic field and the flux due to a cylindrical magnet and reduced it to the computation of elliptical integrals that could be calculated using Mathematica. However, because of the strong similarity with electrostatics, we find that the scalar potential method is much more accessible to undergraduate students. In addition, by choosing to keep an appropriate number of terms in Eq. (13), students can compute the scalar potential to any desired level of accuracy.

\section{COMPUTATION OF FLUX}

As the magnet travels through the copper pipe, the changing magnetic flux causes eddy currents to form in the pipe. We will assume that the pipe thickness is small compared to the radius of the pipe (Refs. 14, 15, and 17 have studied the effect of thickness more carefully). We also assume that the magnet falls coaxially through the conducting pipe, and thus an azimuthal symmetry is maintained throughout the motion. In this case, the eddy currents generated in the pipe would form perfect circles perpendicular to the axis of symmetry. Using the magnetic fields given in Eqs. (14) or (15), we will now determine the flux through a circular cross-section of the pipe. We first compute the flux generated by a single disk and then compute the total flux from the magnet by combining the flux from two disks.

To compute the flux through a circular loop of (average) radius $R_{p}$ a distance $z$ from the disk, we choose a spherical surface, whose center lies at the center of one of the disks of the magnet (see Fig. 5). This particular choice simplifies our calculations considerably. The normal component of the $\vec{B}$ field on this surface is simply the radial derivative of $\mu_{0} \Psi_{m}$. More importantly, because $R_{p}>R_{m}$, independent of the value of $z$, the distance of every point of this spherical

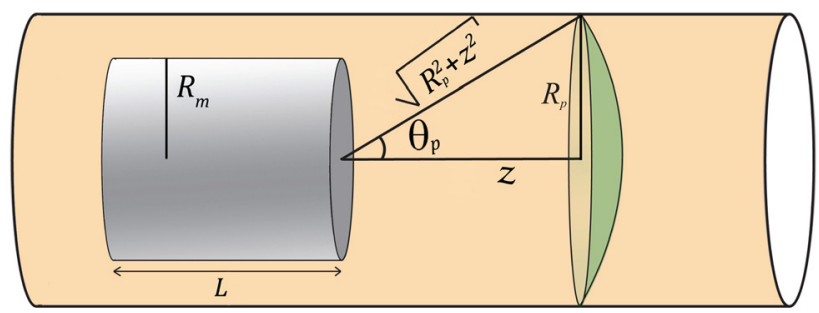

Fig. 5. Schematic diagram showing the magnet in the pipe and the spherical surface used to compute the flux. For our setup, we have $R_{m}=0.0127 \mathrm{~m}$ and $R_{p}=0.01365 \mathrm{~m}$, while the thickness of the pipe is $0.00127 \mathrm{~m}$. surface is larger than the radius of the (magnetically) charged disk. This is the reason we were able to set all the $a_{\ell}$ 's equal to zero in Eq. (9).

Integrating over the spherical surface shown in Fig. 5, the flux $\Phi_{m}(z)$ through a circular loop a distance $z$ from the front disk is given by

$$
\begin{aligned}
\Phi_{m}(z) & =\int_{S} \vec{B} \cdot \hat{r} d a=-\mu_{0} \int_{S} \frac{\partial \Psi_{m}(r, \theta)}{\partial r} d a \\
& =\left.\sum_{\ell=0}^{\infty} b_{2 \ell} \frac{\partial}{\partial r}\left(\frac{1}{r^{2 \ell+1}}\right)\right|_{r=\sqrt{R_{p}^{2}+z^{2}}} \int_{0}^{\theta_{p}} \mathcal{P}_{2 \ell}(\cos \theta) \sin \theta d \theta d \phi \\
& =2 \pi \mu_{0} \sum_{\ell=0}^{N} \frac{b_{2 \ell}(2 \ell+1)}{\left(R_{p}^{2}+z^{2}\right)^{\ell}} \int_{u_{p}}^{1} \mathcal{P}_{2 \ell}(u) d u
\end{aligned}
$$

where in the last line we defined $u=\cos \theta$ and $u_{p}=z / \sqrt{R_{p}^{2}+z^{2}}$. We can compute the integral in Eq. (16) using the identity $\mathcal{P}_{2 \ell}(u)=\left[\mathcal{P}_{2 \ell+1}^{\prime}(u)-\mathcal{P}_{2 \ell-1}^{\prime}(u)\right] /(4 \ell+1)$, giving a flux

$$
\begin{aligned}
\Phi_{m}(z)= & \pi \mu_{0} M_{0} R_{m}^{2}\left[1-\frac{z}{\sqrt{R_{p}^{2}+z^{2}}}\right] \\
& +2 \pi \mu_{0} \sum_{\ell=1}^{N} \frac{(2 \ell+1) b_{2 \ell}}{\left(R_{p}^{2}+z^{2}\right)^{\ell}}\left[\frac{1}{4 \ell+1}\right] \\
& \times\left[\mathcal{P}_{2 \ell-1}\left(\frac{z}{\sqrt{R_{p}^{2}+z^{2}}}\right)-\mathcal{P}_{2 \ell+1}\left(\frac{z}{\sqrt{R_{p}^{2}+z^{2}}}\right)\right],
\end{aligned}
$$

where we have substituted the value of $b_{0}$ in the first term. The above expression for $\Phi_{m}(z)$ gives the flux due to a single disk. To compute the flux due to the entire magnet, we need to consider two disks with magnetic charge densities $\pm M_{0}$ separated by a distance $L$. The net flux is then the sum of the contributions from these two disks. In Fig. 6, we plot the magnetic flux through a circular cross-section of the pipe a distance $z$ from the center of the magnet, as well as the flux contributions from $\mu_{0} \vec{H}$ and $\mu_{0} \vec{M}$. Note that a superposition of the $\mu_{0} \vec{H}$ and $\mu_{0} \vec{M}$ contributions gives the magnetic flux, as expected.

\section{COMPUTATION OF EMF}

In order to compute the emf through a circular crosssection of the conducting pipe a distance $z$ from the center of the magnet, we need to determine the change in flux through the loop during a time interval $\Delta t$. We take the $z$-direction pointing up and assume the magnet falls with a constant velocity $\vec{v}=(d z / d t) \hat{z}=-v_{0} \hat{z}$. The emf is then given by

$$
\mathcal{E}=\oint \vec{E} \cdot d \vec{\ell}=-\frac{d \Phi_{m}}{d t}=-\frac{d \Phi_{m}}{d z} \frac{d z}{d t}=v_{0} \frac{d \Phi_{m}}{d z}
$$

The electric field in the wall of the pipe is therefore $E_{\phi}(r)=\mathcal{E} / 2 \pi r$, where $r$ is the distance from the axis of the pipe. Hence, the current density in the pipe is given by $J_{\phi}=\sigma_{c} E_{\phi}=\sigma_{c} \mathcal{E} / 2 \pi r$, where $\sigma_{c}$ is the conductivity of the pipe (in this case $\sigma_{c}=4.91 \times 10^{7} \mathrm{~S} / \mathrm{m}$ for copper). The current $\Delta I$ through a section of the pipe of thickness $d r$ and length $\Delta z$ can then be calculated as 

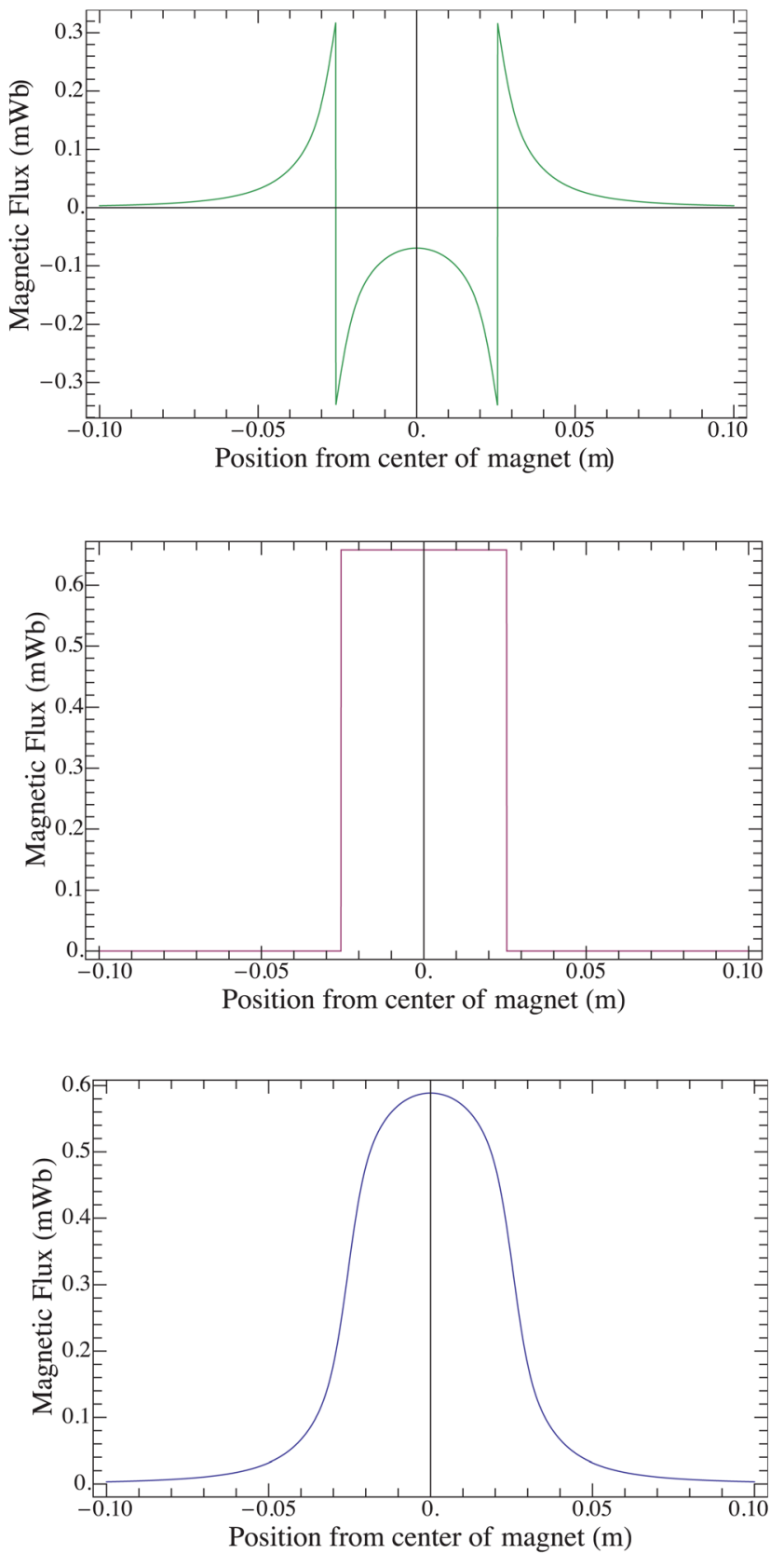

Fig. 6. Contributions of the various fields to the flux $\Phi_{m}(z)$ through a cross-section of the pipe a distance $z$ from the center of the magnet. Contributions are from $\mu_{0} \vec{H}$ (top), $\mu_{0} \vec{M}$ (middle), and $\vec{B}$ (bottom) for our magnet of length $L=5.1 \mathrm{~cm}$.

$$
\begin{aligned}
\int d I & =\int_{R_{1}}^{R_{2}} J_{\phi}(r) d r \Delta z=\frac{v_{0} \sigma_{c}}{2 \pi} \ln \left(\frac{R_{2}}{R_{1}}\right) \frac{d \Phi_{m}}{d z} \Delta z \\
& =\frac{v_{0} \sigma_{c}}{2 \pi} \ln \left(\frac{2 R_{p}+\delta}{2 R_{p}-\delta}\right) \frac{d \Phi_{m}}{d z} \Delta z,
\end{aligned}
$$

where $R_{1}$ and $R_{2}$ are the inner and outer radii of the pipe, $\delta=R_{2}-R_{1}$ is the pipe thickness, and $R_{p}=\left(R_{1}+R_{2}\right) / 2$ is the average radius of the pipe. In our case $\delta / 2 R_{p} \approx 0.04 \ll 1$, so we can expand the logarithm in powers of $\delta / R_{p}$ to get

$$
\begin{aligned}
\Delta I & =\frac{v_{0} \sigma_{c}}{2 \pi}\left(\frac{\delta}{R_{p}}+\frac{1}{12} \frac{\delta^{3}}{R_{p}^{3}}+\cdots\right) \frac{d \Phi_{m}}{d z} \Delta z \\
& \approx \frac{v_{0} \sigma_{c}}{2 \pi} \frac{\delta}{R_{p}} \frac{d \Phi_{m}}{d z} \Delta z,
\end{aligned}
$$

to lower order. Therefore, the total current through a section of the pipe from $z_{1}$ to $z_{2}$ is given by

$$
I=\frac{v_{0} \sigma_{c} \delta}{2 \pi R_{p}}\left[\Phi_{m}\left(z_{2}\right)-\Phi_{m}\left(z_{1}\right)\right]
$$

Verifying this expression for current was a difficult task. We removed a small cylindrical slice (a few millimeters in length) from the middle of the pipe and replaced it with a loop of very thin magnetic wire in series with a large resistor. The gap was kept small to ensure that the velocity of the magnet remained constant. By finding the voltage across the resistor (measured in $\mu \mathrm{V}$ ), we indirectly measured the current in the loop and inferred what the current would have been in the absence of the resistor. Figure 7 shows the current generated in a loop as the magnet passes through it, along with our theoretical prediction. Here, we see that there is reasonably good agreement between the positions of the peaks, but less so for the heights. This discrepancy is caused by the uncertainty in measuring very low voltages. Fortunately - as demonstrated in Sec. VI-the retarding force is much easier to measure than the induced current, so we will obtain much better agreement between theory and experiment when investigating the force as a function of velocity.

\section{COMPUTATION OF RETARDING FORCE}

Because the magnet travels with a constant velocity $\vec{v}$, conservation of energy stipulates that the (thermal) energy loss in the conducting pipe per unit time will be equal to $|\vec{F} \cdot \vec{v}|$, where $\vec{F}$ is the magnetic retarding force. Thus, if we know the power loss and the magnet velocity we can determine the retarding force. To compute the power loss in the pipe, we first determine the differential loss over an infinitesimal length $\Delta z$ of the pipe. This loss is given by

$$
\begin{aligned}
d P & =(d I)^{2}(d R)=\left(J_{\phi} \delta \Delta z\right)^{2} \times \text { Resistance of length } d z \\
& =\left(\frac{v_{0} \sigma_{c} \delta}{2 \pi R_{p}} \frac{d \Phi_{m}}{d z} \Delta z\right)^{2} \frac{2 \pi R_{p}}{\sigma_{c} \delta \Delta z} \\
& =v_{0}^{2} \frac{\sigma_{c} \delta}{2 \pi R_{p}}\left(\frac{d \Phi_{m}}{d z}\right)^{2} \Delta z
\end{aligned}
$$

Assuming the pipe to be much longer than the magnet, we find that the total power loss is given by

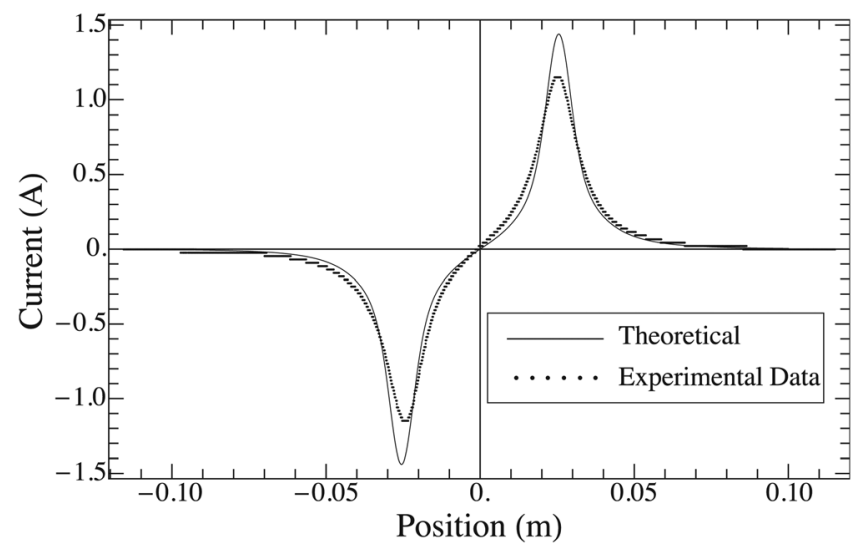

Fig. 7. Theoretical (solid) and experimental (dots) results for the induced current in a loop of wire as our magnet passes through. 


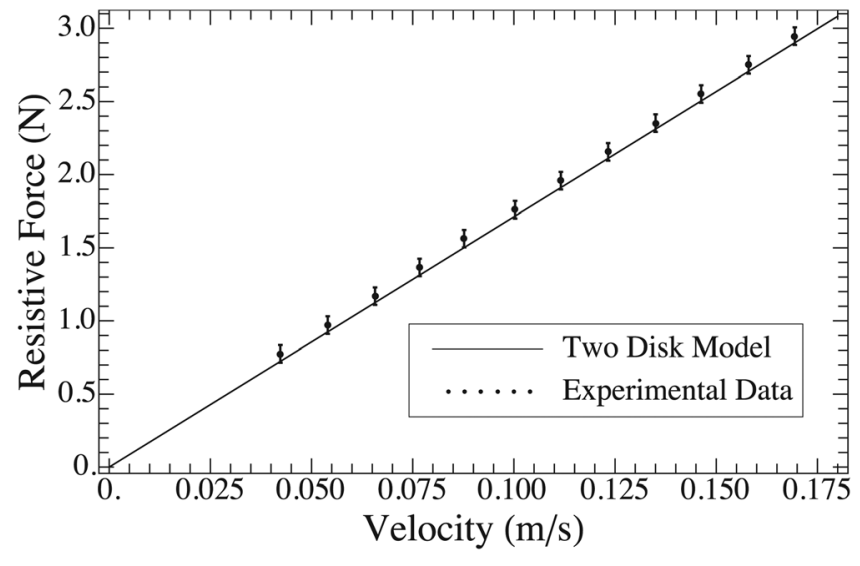

Fig. 8. Experimental (dots) and theoretical (solid) values of the retarding force for various terminal speeds.

$$
\begin{aligned}
P & =v_{0}^{2} \frac{\sigma_{c} \delta}{2 \pi R_{p}} \int_{-\infty}^{\infty}\left(\frac{d \Phi_{m}}{d z}\right)^{2} d z \\
& =2 v_{0}^{2} \frac{\sigma_{c} \delta}{2 \pi R_{p}} \int_{0}^{\infty}\left(\frac{d \Phi_{m}}{d z}\right)^{2} d z
\end{aligned}
$$

Since the dissipated power is given by $P=|\vec{F} \cdot \vec{v}|=F v_{0}$, the retarding force is found to be

$$
F=2 \frac{v_{0} \sigma_{c} \delta}{2 \pi R_{p}} \int_{0}^{\infty}\left(\frac{d \Phi_{m}}{d z}\right)^{2} d z
$$

Therefore, we find that the retarding force is proportional to $v_{0}, \sigma_{c}$, and $\delta$. In particular, if all other parameters are kept fixed, we find that $F \propto v_{0}$. Figure 8 shows the prediction of Eq. (24) compared to the experimental data; apart from a small offset (explained below), the agreement is impressive. Such linear behavior between speed and retarding force has been shown to be an excellent model for speed less than $25 \mathrm{~m} / \mathrm{s}^{14}$

As mentioned, the electrical conductivity of the copper pipe is $\sigma_{c}=4.97 \times 10^{7} \mathrm{~S} / \mathrm{m}$. We carried out an extensive measurement procedure to determine the resistance of copper pipes of various lengths using the 4-point method and Kelvin clips as suggested in Ref. 25. We also contacted the manufacturer of the pipe and the American Society for Testing and Materials (ASTM) for further verification. As seen in Fig. 8, the slopes of the theoretical prediction and the experimental data are almost identical; however, the experimental data are slightly offset from the theoretical prediction such that a best-fit line would not pass through the origin. This discrepancy is caused by a very small, but finite, frictional force in the smart pulleys we used. The total mass hung from these pulleys - the magnet, the weight, and the counter weight - were on the order of $600 \mathrm{~g}$, giving an effective frictional force of about the weight of six grams. ${ }^{26} \mathrm{We}$ emphasize that the vertical offset of $0.0526 \mathrm{~N}$ in Fig. 8 can be accounted for by this frictional force.

\section{CONCLUSION}

We studied the effect of a cylindrical neodymium magnet moving along the axis of a cylindrical conducting pipe. Using the symmetry of the setup and the near uniformity of the magnetization of a neodymium magnet, we were able to develop an analytical model for the induced surface current density and resulting retarding force. The analytically predicted current distribution and the retarding force show excellent agreement with experimental observation. Because we were able to use the magnetic scalar potential technique, which bears a strong resemblance to electrostatics, our analysis is comparatively more accessible to undergraduates than other approaches. In addition, students can compute the flux to any desired level of accuracy by keeping a sufficiently large number of terms in the expansion of the scalar potential.

\section{ACKNOWLEDGMENT}

Two of the authors (B.I. and M.K.) would like to thank Loyola University Chicago for the Mulcahy scholarship, which helped make their undergraduate research possible. A.G. would like to thank the Center for Experiential Learning at Loyola University Chicago for an Engaged Learning Faculty Fellowship that provided partial support for this research. The authors would also like to thank Christopher Kabat for his help in designing the experiments. The authors also acknowledge two anonymous referees, whose detailed comments challenged us to carry out an extensive analysis of sheet resistance to determine the conductivity of the pipe and to conduct a comparative study of various competing models, which provided additional support to our analysis.

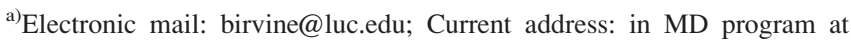
Univ. of Wisconsin, Madison, Wisconsin

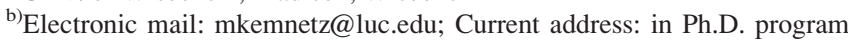
in Aeronautical Engineering at Notre Dame University, Notre Dame, Indiana

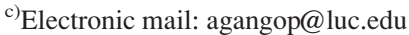

${ }^{d)}$ Electronic mail: truubel@luc.edu

${ }^{1}$ H. D. Wiederick, N. Gauthier, D. A. Campbell, and P. Rochon, "Magnetic braking: Simple theory and experiment," Am. J. Phys. 55, 500-503 (1987).

${ }^{2}$ M. A. Heald, "Magnetic braking: Improved theory," Am. J. Phys. 56, 521-522 (1988).

${ }^{3}$ Jhules A. M. Clack and Terrence P. Toepker, "Magnetic induction experiment," Phys. Teach. 28, 236-238 (1990).

${ }^{4}$ Y. Levin, F. L. da Silveira, and F. B. Rizzato, "Electromagnetic braking: A simple qualitative model," Am. J. Phys. 74, 815-817 (2006).

${ }^{5} \mathrm{~J}$. Íñiguez and V. Raposo, "Measurement of conductivity in metals: A simple laboratory experiment on induced currents," Eur. J. Phys. 28, 1125-1129 (2007).

${ }^{6} \mathrm{~J}$. Íñiguez and V. Raposo, "Comment on 'Magnetic Breaking Revisited: Activities for Undergraduate Laboratory," ” Eur. J. Phys. 30, L19-L21 (2007). ${ }^{7} \mathrm{G}$. Ireson and J. Twidle, "Magnetic braking revisited: Activities for the undergraduate laboratory,” Eur. J. Phys. 29, 745-751 (2008).

${ }^{8}$ M. Marcuso, R. Gass, D. Jones, and C. Rowlett, "Magnetic drag in the quasi-static limit: A computational method," Am. J. Phys. 59, 1118-1123 (1991).

${ }^{9}$ M. Marcuso, R. Gass, D. Jones, and C. Rowlett, "Magnetic drag in the quasi-static limit: Experimental data and analysis," Am. J. Phys. 59, 1123-1129 (1991).

${ }^{10}$ C. MacLatchy, P. Backman, and L. Bogan, "A quantitative magnetic braking experiment," Am. J. Phys. 61, 1096-1101 (1993).

${ }^{11} \mathrm{~L}$. McCarthy, "On the electromagnetically damped mechanical harmonic oscillator,” Am. J. Phys. 64, 885-891 (1996).

${ }^{12}$ J. M. Aguirregabiria, A. Hernandez, and M. Rivas, "Magnetic braking revisited," Am. J. Phys. 65, 851-856 (1997).

${ }^{13} \mathrm{~J}$. Íñiguez et al., "Study of the conductivity of a metallic tube by analyzing the damped fall of a magnet," Eur. J. Phys. 25, 593-604 (2004).

${ }^{14}$ M. Hossein Partovi and E. Morris, "Electrodynamics of a magnet moving through a conducting pipe," Can. J. Phys. 84, 253-274 (2006).

${ }^{15}$ B. Knyazev et al., "Braking of a magnetic dipole moving with an arbitrary velocity through a conducting pipe,” Phys. Usp. 49, 937-946 (2006). 
${ }^{16}$ Jae-Sung Bae, Jai-Hyuk Hwang, Jung-Sam Park, and Dong-Gi Kwag, "Modeling and experiments on eddy current damping caused by a permanent magnet in a conductive tube," J. Mech. Sci. Tech. 23, 3024-3035 (2009).

${ }^{17}$ G. Donoso, C. Ladera, and P. Martin, "Magnet fall inside a conductive pipe: Motion and the role of the pipe wall thickness," Eur. J. Phys. 30, 855-869 (2009).

${ }^{18}$ G. Donoso, C. Ladera, and P. Martin, "Damped fall of magnets inside a conducting pipe," Am. J. Phys. 79, 193-200 (2011).

${ }^{19}$ N. Derby and S. Olbert, "Cylindrical magnets and ideal solenoids," Am. J. Phys. 78, 229-235 (2010).

${ }^{20}$ P. J. Salzman, J. R. Burke, and S. M. Lea, "The effect of electric fields in a classic introductory physics treatment of eddy current forces," Am. J. Phys. 69, 586-590 (2001).

${ }^{21}$ K. D. Hahn, E. M. Johnson, A. Brokken, and S. Baldwin, "Eddy current damping of a magnet moving through a pipe," Am. J. Phys. 66, 1066-1076 (1998).
${ }^{22}$ A physical dipole consists of two magnetic charges $\left( \pm q_{m}\right)$ of opposite polarity separated by a distance $\ell_{m}$. The magnetic field due to a monopole is assumed to be $\mu_{0} q_{m} / 4 \pi r^{2}$. The magnetic charge $q_{m}$ and the separation $\ell_{m}$ are determined by stipulating that the dipole moment $\left(q_{m} \ell_{m}\right)$ be equal to that of our magnet $\left(\pi R_{m}^{2} L M_{0}\right)$. Additionally, we demand that the magnetic field on the axis of two monopoles situated at $z= \pm \ell_{m} / 2$ has a best fit with the experimental data. This generates a best estimate for $\ell_{m}$ of $3.245 \mathrm{~cm}$.

${ }^{23}$ John D. Jackson, Classical Electrodynamics, 3rd ed. (John Wiley \& Sons, Inc., New York, 1999), pp. 154-155.

${ }^{24}$ For more information on Legendre Polynomials and their properties, see George B. Arfken, Hans J. Weber, and Frank E. Harris, Mathematical Methods for Physicists, 7th ed. (Academic Press, Waltham, MA, 2013), pp. 715-772.

${ }^{25}$ F. M. Smits, "Measurement of sheet resistivities using four point probe," Bell Syst. Tech. J. 37, 711-718 (1958).

${ }^{26} \mathrm{We}$ computed this frictional force by forming an Atwood machine type experiment with varying $\Delta m$, but keeping $m_{1}+m_{2}=600 \mathrm{~g}$.

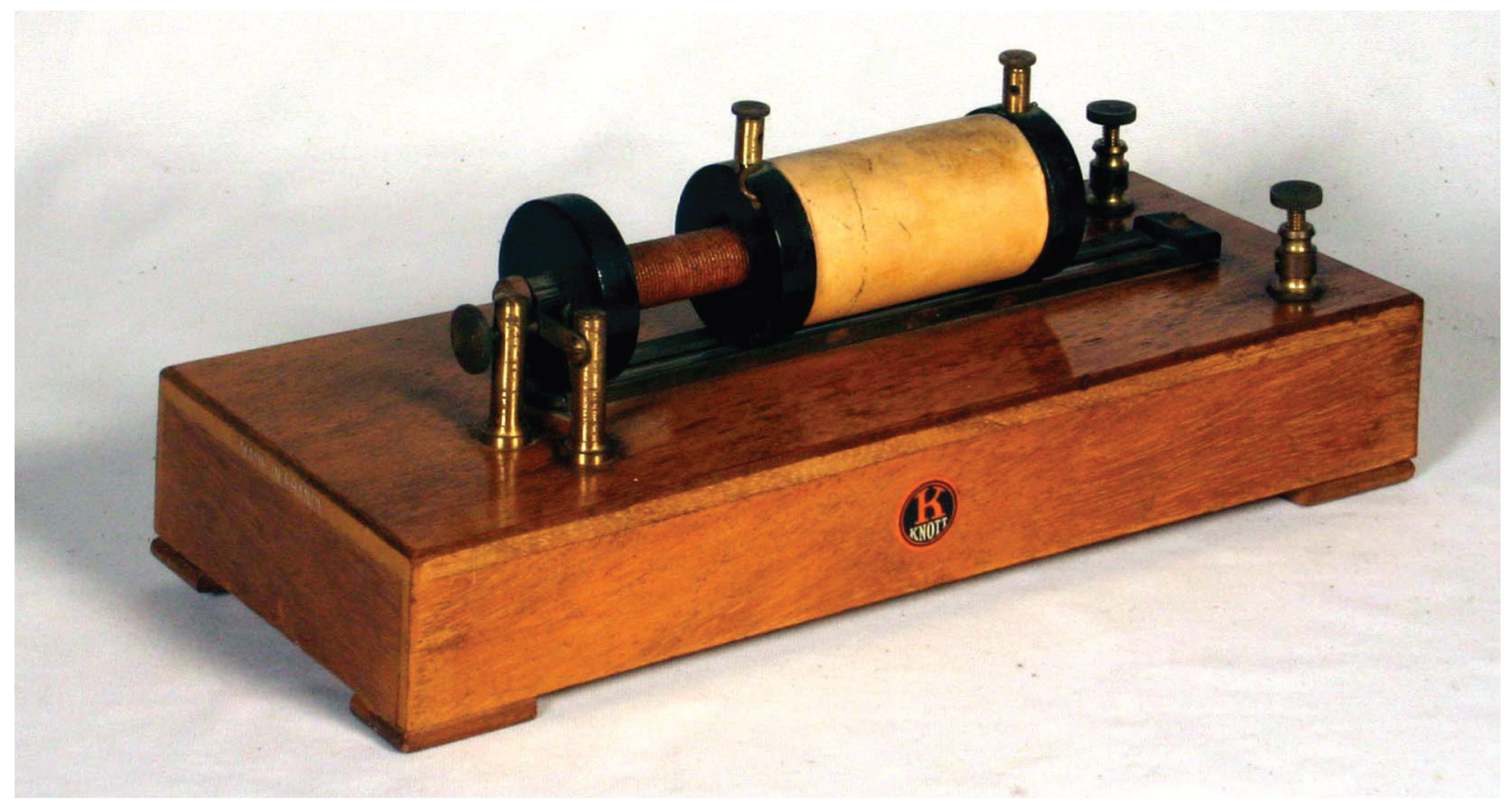

\section{Demonstration Induction Coil}

From the 1916 catalogue of the L. E. Knott Apparatus Company: "This type of coil is of value in the study of the office of the primary in induction coil construction. Both primary and secondary are mounted on a polished mahogany box, but the mount is designed so that the secondary may be adjusted to enclose varying amounts of the primary. This instrument is handsomely finished, all brass parts polished and lacquered, and will give a $1 / 4$-inch spark...\$5.00" This instrument is on long term loan to the Greenslade Collection from the Appalachian State University Physics Department. (Notes and photograph by Thomas B. Greenslade, Jr., Kenyon College) 